Document downloaded from:

http://hdl.handle.net/10251/50301

This paper must be cited as:

Company Rossi, R.; Jódar Sánchez, LA.; Pintos Taronger, JR. (2012). A consistent stable numerical scheme for a nonlinear option pricing model in illiquid markets. Mathematics and Computers in Simulation. 82(10):1972-1985. doi:10.1016/j.matcom.2010.04.026.

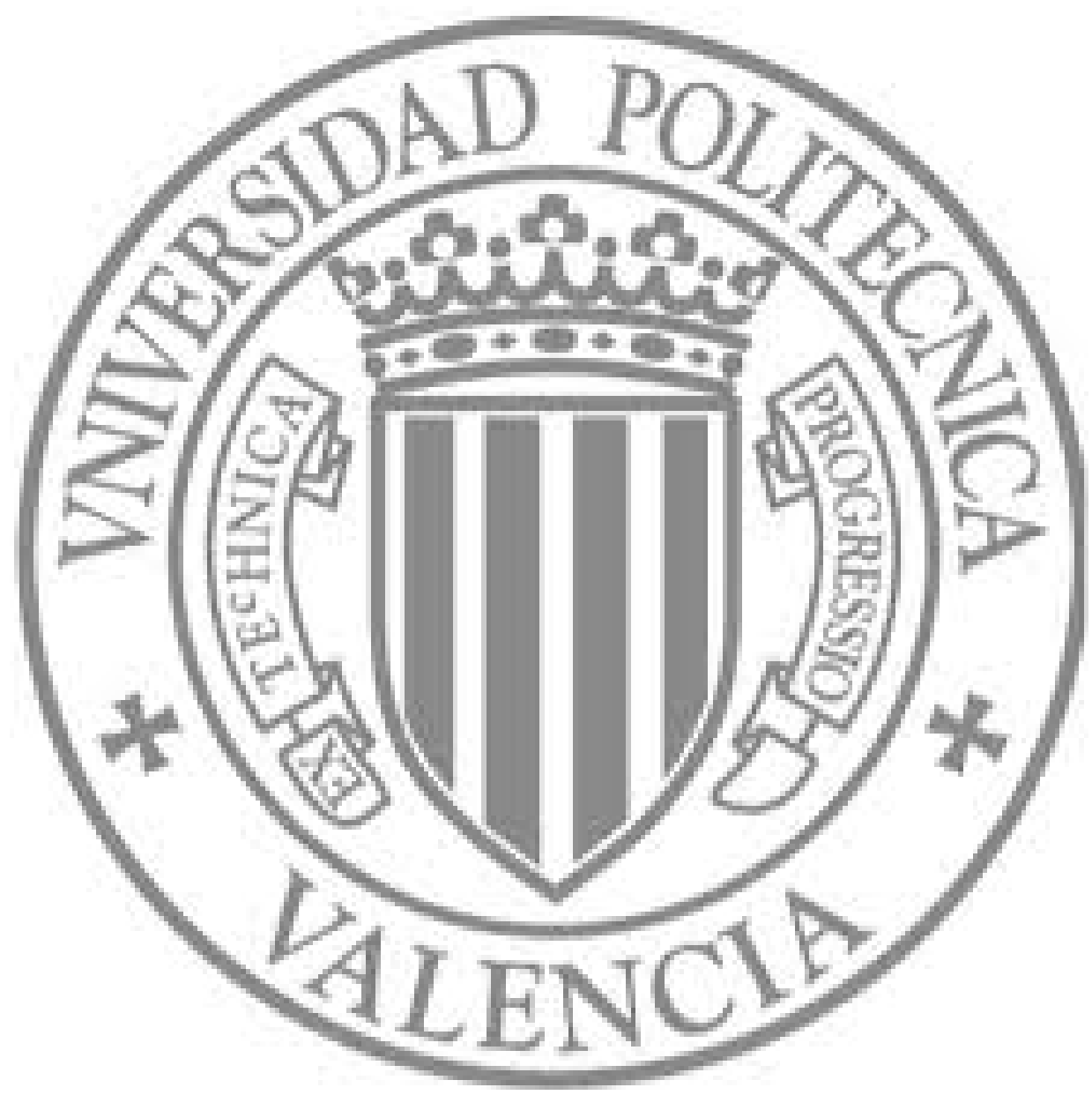

The final publication is available at

http://dx.doi.org/10.1016/j.matcom.2010.04.026

Copyright Elsevier 


\title{
A consistent stable numerical scheme for a nonlinear option pricing model in illiquid markets.
}

\author{
Rafael Company ${ }^{1,}$, Lucas Jódar \\ José-Ramón Pintos \\ Instituto de Matemática Multidisciplinar, Universidad Politécnica de Valencia, Camino \\ de Vera s/n, 46022 Valencia, España
}

\begin{abstract}
Markets liquidity is an issue of very high concern in financial risk management. In a perfect liquid market the option pricing model becomes the well-known linear Black-Scholes problem. Nonlinear models appear when transaction costs or illiquid markets effects are taken into account. This paper deals with the numerical analysis of nonlinear Black-Scholes equations modeling illiquid markets when price impact in the underlying asset market affects the replication of a European contingent claim. Numerical analysis of a nonlinear model is necessary because disregarded computations may waste a good mathematical model. In this paper we propose a finite-difference numerical scheme that guarantees positivity of the solution as well as stability and consistency.
\end{abstract}

Key words: Nonlinear Numerical Analysis, Simulation, Option Pricing, Illiquid Markets

\section{Introduction}

An option is a financial contract entered into by two parties, a buyer and a seller. The buyer of the contract obtains the right to trade an underlying asset, such as a stock, for a specified price, called the strike, on or before a maturity date. Options providing the right to buy the underlying asset are

Email addresses: rcompany@imm.upv.es (Rafael Company), ljodar@imm.upv.es (Lucas Jódar), jrpt60@gmail.com (José-Ramón Pintos) 
known as calls, whereas options conferring the right to sell the underlying asset are referred to as puts.

There are many varieties of options. European options may only be exercised on the maturity date. American options may be exercised any time up to and including the maturity date, see [12] for details.

One of the modern financial theory's biggest successes in terms of both approach and applicability has been Black-Scholes pricing, which allows investors to calculate the 'fair' price of a derivative security whose value depends on the value of another security, known as the underlying, based on a small set of assumptions on the price behaviour of that underlying. One of the major assumptions of Black-Scholes model is that the market in the underlying asset is perfectly elastic so that large trades do not affect prices in equilibrium. This occurs in perfectly liquid markets, but the case is clearly unrealistic.

The presence of price impact of investors' trading has been widely documented and extensively analyzed in the literature, see, for instance, [20, 13]. In the presence of asymmetric information, [15] and [2] use an equilibrium approach to investigate how informed traders reveal information and affect the market price through trading. [23] studies a dynamic model of a financial market with a large trader who does not have any private information on the asset value but trades only to share risk. He shows that the equilibrium stock price is linear in the investor's order size.

Assuming that price impact depends only on the total wealth and the position of a trader but not on how she trades, illiquid problem has been treated in $[6,7,17]$.

Sircar and Papanicolau [21] use an economic model consisting of two distinct groups of traders that accounts for the feedback effect from the Black-Scholes dynamic hedging strategies of the price of the asset.

In [10] and [11], the authors study how the hedging strategy affects the price of the underlying security. An interesting paper, closely related to the last references is [16], where the authors examine how price impact in the underlying asset market affects the replication of a European contingent claim. They obtain a generalized Black-Scholes pricing PDE that for the case where interest rate $(r)$ and the reference volatility $(\sigma>0)$ are constant, takes the form 


$$
\begin{gathered}
\frac{\partial v}{\partial t}(S, t)+\frac{\sigma^{2} S^{2}}{2\left(1-\lambda(S, t) S \frac{\partial^{2} v}{\partial S^{2}}(S, t)\right)^{2}} \frac{\partial^{2} v}{\partial S^{2}}(S, t)+r S \frac{\partial v}{\partial S}(S, t)-r v(S, t)=0 \\
(S, t) \in] 0,+\infty[\times] 0, T] \\
v(S, T)=f(S), \quad 0<S<+\infty
\end{gathered}
$$

where v is the price of the option depending on the underlying asset $S$ and the time $t . T$ is the maturity and $\frac{\partial v}{\partial S}, \frac{\partial^{2} v}{\partial S^{2}}$ are called the Greeks Delta and Gamma of the option respectively. [16] establish the existence and uniqueness of a classical solution to this PDE, for the case where the payoff function $f(S)$ satisfies:

$f\left(e^{x}\right)$ is Lipschitz continuous and $e^{-\alpha \sqrt{1+x^{2}}} f\left(e^{x}\right)$ is bounded for some $\alpha \geq 0$.

This condition is satisfied when the payoff function of the European contingent claim is a continuous piecewise linear function. In $(1.1), \lambda(S, t)$ is the price impact factor of the trader involved in the hedging strategies influence, and that satisfies some regularity conditions included in Theor. 1 of [16].

Note that considering the change $\tau=T-t, u(S, \tau)=v(S, t)$, problem (1.1)-(1.2) takes the form

$$
\left.\begin{array}{r}
\frac{\partial u}{\partial \tau}-\frac{\sigma^{2} S^{2}}{2\left(1-\lambda(S, T-\tau) S \frac{\partial^{2} u}{\partial S^{2}}\right)^{2}} \frac{\partial^{2} u}{\partial S^{2}}-r S \frac{\partial u}{\partial S}+r u=0 \\
(S, t) \in] 0,+\infty[\times] 0, T], \\
u(S, 0)=f(S), \quad 0<S<+\infty
\end{array}\right\}
$$

In this paper we are interested in the numerical analysis of problem (1.3) for a continuous piecewise linear payoff function $f(S)$ and $\lambda(S, t)$ is the function used in [16] and consistent with the price impact form obtained in $[2,3,15,23]$

$$
\lambda(S, t)= \begin{cases}\frac{\gamma}{S}\left(1-e^{-\beta(T-t)}\right) & \text { if } \underline{S} \leq S \leq \bar{S} \\ 0 \quad, & \text { otherwise, }\end{cases}
$$


where the constant price impact coefficient $\gamma>0$ measures the price impact per traded share, and $\underline{S}$ and $\bar{S}$ represent respectively, the lower and upper limit of the stock price within which there is a price impact. This form assumes that as a trader buys, the stock price goes up and as he/she sells, the stock price goes down. Although to fix ideas we take the above mentioned function $\lambda(S, t)$, it is important to point out that conclusions remain true for a general $\lambda(S, t)$ function that could be approximated arbitrarily well by a smooth function satisfying the regularity conditions of theorem 1 of [16].

Some authors $[1,8]$, dealing with nonlinear option pricing problems and using linearization techniques, show that resulting linearized problem is stable. Implicit numerical schemes for nonlinear option pricing PDEs with uncertain volatility have been analyzed in [18] where an iterative approach is required to solve the nonlinear algebraic equations resulting from the discretization. Implicit-Explicit (IMEX) Runge-Kutta methods have been proposed in [4] for solving Parabolic Integro-Differential equations arising in JumpDiffusion models for option pricing. In [24] and [25] the author presents a transformation technique that can be used in analysis and numerical computation of the early exercise boundary for an American style of vanilla options that can be modelled by class of generalized Black-Scholes equations.

With respect to the numerics of the illiquid marked problem (1.3), in [16] the authors make numerical simulations but do not provide any information about the relationship between stepsize discretized variables in order to guarantee reliable results like positivity and stability conditions, see [19] . Careless numerical computation may waste a good mathematical model and once one uses a numerical scheme, stability and consistency must be treated. For instance, a scheme may be consistent with an equation and inconsistent with another. In [5], consistent and stable numerical schemes for nonlinear option pricing PDE problems have been recently proposed.

This paper is organized as follows. Section 2 deals with the construction of an explicit numerical scheme for problem (1.3) that is easy to implement and has nice monotonicity properties. Because of its influence in the nonlinearity of the PDE problem, the numerical behaviour of the Gamma of the option $\frac{\partial^{2} u}{\partial S^{2}}$ is studied. Monotonicity properties of the introduced numerical scheme are studied in section 3 in order to guarantee positivity and stability of the numerical solution of problem (1.3). In section 4, the consistency of the numerical scheme with equation of problem (1.3) is treated. Finally, section 5 contains several examples and simulations. For the sake of clarity in the 
presentation we recall that a real function $f(x)$ is said to be convex in an interval $I$ if

$$
f(\alpha x+\beta y) \leq \alpha f(x)+\beta f(y),
$$

for $0 \leq \alpha, \beta \leq 1, \quad \alpha+\beta=1$, for $x, y$ lying in $I$.

\section{Numerical scheme construction}

As the domain of problem (1.3) is unbounded in the underlying asset variable $S$, we need to bound such domain in order to compute a numerical solution. The bounded numerical domain can be chosen according with different criteria, see [14] and [9] for instance. Let us denote $[0, b]$ the domain for the asset variable $S$, where $b$ is chosen so that the interval includes the price impact, $0<\underline{S}<\bar{S}<b$. Thus, the numerical domain is $(S, \tau) \in[0, b] \times[0, T]$ and the nodes $S_{j}=j h, h=\triangle S, \tau^{n}=n k, k=\triangle \tau$, with $0 \leq j \leq N$, $0 \leq n \leq l, N h=b$ and $l k=\tau$.

Let us introduce the approximations of the partial derivatives and the operators $\nabla_{j}^{n}$ and $\triangle_{j}^{n}$ :

$$
\begin{aligned}
& U_{j}^{n} \simeq u\left(S_{j}, \tau^{n}\right), \quad \frac{\partial u}{\partial \tau}\left(S_{j}, \tau^{n}\right)=\frac{U_{j}^{n+1}-U_{j}^{n}}{k}+O(k), \\
& \frac{\partial u}{\partial S}\left(S_{j}, \tau^{n}\right)=\frac{U_{j+1}^{n}-U_{j-1}^{n}}{2 h}+O\left(h^{2}\right)=\nabla_{j}^{n}(U)+O\left(h^{2}\right), \\
& \frac{\partial^{2} u}{\partial S^{2}}\left(S_{j}, \tau^{n}\right)=\frac{U_{j-1}^{n}-2 U_{j}^{n}+U_{j+1}^{n}}{h^{2}}+O\left(h^{2}\right)=\triangle_{j}^{n}(U)+O\left(h^{2}\right) .
\end{aligned}
$$

Note that due to the use of centered approximations of the derivatives, at $S_{0}=0$ and $S_{N}=b$, there appear external fictitious nodes $S_{-1}=-h$ and $S_{N+1}=(N+1) h$. The approximations $U_{-1}^{n}$ and $U_{N+1}^{n}$ are obtained by using linear interpolation throughout the approximations obtained in the closest interior nodes of the numerical domain.

Thus

$$
U_{-1}^{n}=2 U_{0}^{n}-U_{1}^{n}, \quad U_{N+1}^{n}=2 U_{N}^{n}-U_{N-1}^{n}, \quad 0 \leq n \leq l,
$$

and from (2.1) one gets

$$
\triangle_{0}^{n}=\triangle_{N}^{n}=0, \quad 0 \leq n \leq l .
$$


By replacing the partial derivatives of equation (1.3) by the approximations given in (2.1) one gets the numerical scheme at the internal mesh points:

$$
\begin{gathered}
U_{j}^{n+1} \\
=\left(1-k r-\frac{k}{h^{2}} \beta_{j}^{n}\right) U_{j}^{n}+\frac{k}{2 h^{2}}\left[\left(\beta_{j}^{n}-j h^{2} r\right) U_{j-1}^{n}+\left(\beta_{j}^{n}+j h^{2} r\right) U_{j+1}^{n}\right], \\
1 \leq j \leq N-1,
\end{gathered}
$$

where

$$
\begin{aligned}
& \beta_{j}^{n}=\frac{\sigma^{2} S_{j}^{2}}{\left[1-\nu_{j}^{n}\right]^{2}} \geq 0, \quad 0 \leq j \leq N, \quad 0 \leq n \leq l, \\
& \nu_{j}^{n}= \begin{cases}\gamma\left(1-e^{-\beta n k}\right) \triangle_{j}^{n}(U), & \underline{S} \leq S_{j} \leq \bar{S} \\
0, & \text { otherwise. }\end{cases}
\end{aligned}
$$

Using (2.2) and (2.4), at the boundaries we obtain

$$
\begin{gathered}
U_{0}^{n+1}=U_{0}^{n}=\ldots=U_{0}^{0}=f(0), \\
U_{N}^{n+1}=(1+(N-1) k r) U_{N}^{n}-N k r U_{N-1}^{n} .
\end{gathered}
$$

For the sake of the convenience we will write scheme (2.4), (2.7), (2.8) in vector form. Let us denote the vector in $\mathbb{R}^{N+1}$

$$
U^{n}=\left[\begin{array}{llll}
U_{0}^{n} & U_{1}^{n} & \ldots & U_{N}^{n}
\end{array}\right]^{t} ;
$$

and let $A, B(n), C$, and $D$ be the matrices in $\mathbb{R}^{(N+1) \times(N+1)}$ defined by

$$
\begin{gathered}
A=\left[\begin{array}{ccccc}
0 & 0 & 0 & \cdots & 0 \\
1 & -2 & 1 & \cdots & 0 \\
& \ddots & \ddots & \ddots & \\
0 & \cdots & 1 & -2 & 1 \\
0 & \cdots & 0 & 0 & 0
\end{array}\right], C=\left[\begin{array}{ccccc}
-2 & 2 & 0 & \cdots & 0 \\
-1 & 0 & 1 & \cdots & 0 \\
& \ddots & \ddots & \ddots & \\
0 & \cdots & -1 & 0 & 1 \\
0 & \cdots & 0 & -2 & 2
\end{array}\right] \\
B(n)=\operatorname{diag}\left(\beta_{0}^{n}, \ldots, \beta_{N}^{n}\right), \quad D=\operatorname{diag}(0,1,2, \ldots, N) .
\end{gathered}
$$


Hence, scheme (2.4), (2.7), (2.8) can be written in the following explicit vector form

$$
U^{n+1}=\left[(1-k r) I+\frac{k}{2 h^{2}} B(n) A+\frac{k r}{2} D C\right] U^{n}, \quad 0 \leq n \leq l-1,
$$

where $I$ denotes the identity matrix in $\mathbb{R}^{(N+1) \times(N+1)}$, and satisfies the initial condition

$$
U^{0}=\left[\begin{array}{llll}
f(0) & f(h) \ldots f(N h)
\end{array}\right]^{t} .
$$

The strong dependence of the model (1.3) in terms of the Gamma of the option $u_{S S}$ suggests to pay attention to the dynamic behaviour of its numerical approximation $\triangle_{j}^{n}$ that will play an important role in the numerical analysis of the scheme (2.12), (2.13).

Let us denote the vector $\triangle^{n}(U)$ in $\mathbb{R}^{N+1}$ defined by

$$
\triangle^{n}(U)=\left[\triangle_{0}^{n}(U) \triangle_{1}^{n}(U) \ldots \triangle_{N}^{n}(U)\right], \quad 0 \leq n \leq l .
$$

Taking into account (2.1),(2.9),(2.10) and (2.14), one gets

$$
\triangle^{n}(U)=\frac{1}{h^{2}} A U^{n}
$$

and from (2.12),(2.15) it follows that

$$
\begin{aligned}
\triangle^{n+1}(U) & =\frac{1}{h^{2}} A U^{n+1}=\frac{1}{h^{2}} A\left((1-k r) I+\frac{k}{2 h^{2}} B(n) A+\frac{k r}{2} D C\right) U^{n} \\
& =(1-k r) \triangle^{n}(U)+\frac{k}{2 h^{2}} A B(n) \triangle^{n}(U)+\frac{k r}{2 h^{2}} A D C U^{n} .
\end{aligned}
$$

Easy computations yield 
$\mathrm{ADC}$

$=\left[\begin{array}{cccccccc}0 & 0 & 0 & 0 & & & \cdots & 0 \\ 2 & -2 & -2 & 2 & 0 & & \cdots & \vdots \\ -1 & 4 & -2 & -4 & 3 & 0 & \cdots & \vdots \\ 0 & -2 & 6 & -2 & -6 & 4 & \cdots & \vdots \\ \vdots & & \ddots & \ddots & \ddots & \ddots & & \vdots \\ \vdots & -(j-1) & 2 j & -2 & -2 j & j+1 & \vdots \\ \vdots & & \ddots & \ddots & \ddots & \ddots & \ddots & \vdots \\ \vdots & & & -(N-3) & 2(N-2) & -2 & -2(N-2) & N-1 \\ \vdots & & & & -(N-2) & 2(N-1) & -(N+2) & 2 \\ 0 & \ldots & & & 0 & 0 & 0 & 0\end{array}\right]$

From (2.16),(2.17) one gets the componentwise expression of scheme (2.16) where it is important to remark that the dependence on $U_{j}^{n}$ involved in $\frac{k r}{2 h^{2}} A D C U^{n}$ can be expressed in terms of $\triangle_{j}^{n}$ because of

$$
\triangle_{j}^{n}(U)=\frac{U_{j-1}^{n}-2 U_{j}^{n}+U_{j+1}^{n}}{h^{2}}
$$

see $(2.1)$.

Hence, and taking into account (2.3), for $j=0$ and $j=N$, one gets

$$
\triangle_{0}^{n+1}=\triangle_{0}^{n}=0, \quad \triangle_{N}^{n+1}=\triangle_{N}^{n}=0,
$$

and for $1 \leq j \leq N-1$,

$$
\begin{gathered}
\triangle_{j}^{n+1}=\left(1-\frac{k}{h^{2}} \beta_{j}^{n}\right) \triangle_{j}^{n} \\
+\frac{k}{2 h^{2}}\left[\left(\beta_{j-1}^{n}-(j-1) h^{2} r\right) \triangle_{j-1}^{n}+\left(\beta_{j+1}^{n}+(j+1) h^{2} r\right) \triangle_{j+1}^{n}\right] .
\end{gathered}
$$

\section{Monoticity, positivity and stability}

As scheme (2.4) shows, positivity of $U_{j}^{n+1}$ is linked with the behaviour of $\beta_{j}^{n}$ that is related to $\triangle_{j}^{n}$ throughout $(2.5),(2.6)$. 
Let us assume that the payoff function $f(S)$ is convex. Note that for $n=0$, from $(2.1)$,

$$
\triangle_{j}^{0}=\frac{f\left(S_{j-1}\right)-2 f\left(S_{j}\right)+f\left(S_{j+1}\right)}{h^{2}}, 1 \leq j \leq N-1 .
$$

As $f(S)$ is convex, from (1.5), taking $\alpha=\beta=\frac{1}{2}, x=S_{j-1}, y=S_{j+1}$, one gets

$$
f\left(S_{j}\right)=f\left(\frac{1}{2} S_{j-1}+\frac{1}{2} S_{j+1}\right) \leq \frac{1}{2} f\left(S_{j-1}\right)+\frac{1}{2} f\left(S_{j+1}\right) .
$$

From (3.1) and (3.2) it follows that

$$
\triangle_{j}^{0} \geq 0, \quad \forall h>0, \quad 1 \leq j \leq N-1,
$$

and from (2.3),

$$
\triangle_{0}^{0}=\triangle_{N}^{0}=0 .
$$

For each $n$ with $0 \leq n \leq l-1$, let us denote

$$
\sum(n)=\sum_{j=0}^{N} \triangle_{j}^{n}=\sum_{j=1}^{N-1} \triangle_{j}^{n} .
$$

From (2.18), (2.19) and (3.4)

$$
\begin{aligned}
& \sum(n+1)=\sum_{j=1}^{N-1}\left(1-\frac{k}{h^{2}} \beta_{j}^{n}\right) \triangle_{j}^{n} \\
&+\frac{k}{2 h^{2}}\left(\sum_{j=0}^{N-2}\left(\beta_{j}^{n}-j h^{2} r\right) \triangle_{j}^{n}+\sum_{j=2}^{N}\left(\beta_{j}^{n}+j h^{2} r\right) \triangle_{j}^{n}\right) \\
&=\sum(n)-\frac{k}{2 h^{2}}\left[\left(\beta_{1}^{n}+h^{2} r\right) \triangle_{1}^{n}+\left(\beta_{N-1}^{n}-(N-1) h^{2} r\right) \triangle_{N-1}^{n}\right] .
\end{aligned}
$$

The next result shows that numerical solution $U_{j}^{n}$ of scheme (2.4) preserves the convexity with respect to the underlying asset variable along the time and that $\sum(n)$ is decreasing. 
Lemma 1. Let $r$ be the riskless interest rate, $\sigma$ the volatility and $\gamma$ the impact factor parameter of the illiquid market. Assume that the payoff function $f(S)$ is convex and let us denote

$$
\eta=\gamma\left(1-e^{-\beta \tau}\right) \Sigma(0), \quad \tau=l k .
$$

Suppose that $S \in[0, b]$,

$$
\sigma^{2} \geq r \quad, \quad 0 \leq \eta<1
$$

and

$$
\frac{k}{h^{2}} \leq \frac{(1-\eta)^{2}}{\sigma^{2} b}
$$

Then

(i) $\triangle_{j}^{n} \geq 0, \quad O \leq j \leq N, \quad 0 \leq n \leq \ell$.

(ii) $\sum(n+1) \leq \sum(n), \quad 0 \leq n \leq \ell-1$.

Proof. We prove both parts $(i)$ and $(i i)$ using the induction principle. Note that for $n=0$, part $(i)$ is a direct consequence of convexity of $f(S)$, see (3.3).

For $n=0,(3.5)$ takes the form

$$
\sum(1)=\sum(0)-\frac{k}{2 h^{2}}\left[\left(\beta_{1}^{0}+h^{2} r\right) \triangle_{1}^{0}+\left(\beta_{N-1}^{0}-(N-1) h^{2} r\right) \triangle_{N-1}^{0}\right]
$$

From (2.5), (2.6) one gets

$$
\beta_{j}^{0}=\frac{j^{2} h^{2} \sigma^{2}}{\left[1-\nu_{j}^{0}\right]^{2}}=j^{2} h^{2} \sigma^{2}, \quad 0 \leq j \leq N .
$$

From (3.7), (3.9) and (3.10) one gets (ii) for $n=0$. Now we prove that $\triangle_{j}^{1} \geq 0$, for $1 \leq j \leq N-1$. Note that from (2.5) and (3.8)

$$
1-\frac{k}{h^{2}} \beta_{j}^{0} \geq 1-\frac{k}{h^{2}}\left(b^{2} \sigma^{2}\right) \geq 0,
$$

and from (2.19), (3.7) and (3.10), all the coefficients of (2.19) are nonnegative for $n=0$ and thus $\triangle_{j}^{1} \geq 0$ for $1 \leq j \leq N-1$. 
Using induction hypothesis, let us assume that

$$
\sum(n) \leq \sum(n-1), \quad n>1 .
$$

and

$$
\triangle_{j}^{n} \geq 0
$$

From (2.6) and (3.6),

$$
\begin{aligned}
& 0 \leq \nu_{j}^{n} \leq \gamma\left(1-e^{-\beta n k}\right) \triangle_{j}^{n} \leq \gamma\left(1-e^{-\beta n k}\right) \sum(n) \\
& \leq \gamma\left(1-e^{-\beta n k}\right) \sum(0) \leq \eta
\end{aligned}
$$

From (2.5) and (3.8), it follows that

$$
0 \leq \beta_{j}^{n} \leq \frac{\sigma^{2} S_{j}^{2}}{(1-\eta)^{2}} \leq \frac{\sigma^{2} b^{2}}{(1-\eta)^{2}} \leq \frac{h^{2}}{k}
$$

and from $(2.19), \triangle_{j}^{n+1} \geq 0$. Hence $\sum(n+1) \geq 0$.

Note that

$$
\beta_{j}^{n}-j h^{2} r=j h^{2}\left(\frac{\sigma^{2} j}{\left(1-\nu_{j}^{n}\right)^{2}}-r\right) \geq j h^{2}\left(\sigma^{2} j-r\right) \geq 0, \quad 1 \leq j \leq N .
$$

and from (3.5) and (3.16) for $j=N-1$, one concludes that

$$
\sum(n+1) \leq \sum(n) .
$$

Thus the result is established.

For the sake of clarity in the presentation we introduce the definition of a monoticity-preserving scheme.

Definition1. Consider the scheme $F\left(u_{j}^{n}\right)=0, j \in J, n \in L$ where $J$ and $L$ are sets of nonnegative integers. We say that the scheme is monoticitypreserving, if, assuming that

$$
u_{j+1}^{n} \geq u_{j}^{n}, \quad j \in J, \quad j+1 \in J,
$$

then, it occurs that

$$
u_{j+1}^{n+1} \geq u_{j}^{n+1}, \quad j \in J, \quad j+1 \in J .
$$


Theorem 1. Under hypotheses of Lemma 1, let us introduce the positive constant

$$
L(h)=\frac{(1-\eta)^{2}}{\sigma^{2} b^{2}+\frac{1}{2}(1-\eta)^{2} r h^{2}},
$$

and assume that

$$
k \leq h^{2} L(h),
$$

then the numerical scheme (2.4), (2.7), (2.8) is monoticity-preserving, with $0 \leq j \leq N, 0 \leq n \leq \ell$.

Proof. Let us write

$$
\begin{gathered}
u_{j+1}^{n+1}-u_{j}^{n+1} \\
=\left(u_{j+1}^{n+1}-(1-k r) u_{j+1}^{n}\right)+(1-k r)\left(u_{j+1}^{n}-u_{j}^{n}\right)-\left(u_{j}^{n+1}-(1-k r) u_{j}^{n}\right) .
\end{gathered}
$$

By assuming $u_{j+1}^{n} \geq u_{j}^{n}, 0 \leq n \leq \ell-1,1 \leq j \leq N-1$, and from (2.4) it follows that

$$
\begin{aligned}
& u_{j}^{n+1}-(1-k r) u_{j}^{n} \\
& \leq-\frac{k}{h^{2}} \beta_{j}^{n} u_{j}^{n}+\frac{k}{2 h^{2}}\left[\left(\beta_{j}^{n}-j h^{2} r\right) u_{j}^{n}+\left(\beta_{j}^{n}+j h^{2} r\right) u_{j+1}^{n}\right] \\
& \quad=\frac{k}{2 h^{2}}\left(u_{j+1}^{n}-u_{j}^{n}\right)\left(\beta_{j}^{n}+j h^{2} r\right), \quad 1 \leq j \leq N-1,
\end{aligned}
$$

and in an analogous way

$$
u_{j}^{n+1}-(1-k r) u_{j}^{n} \geq \frac{-k}{2 h^{2}}\left(u_{j}^{n}-u_{j-1}^{n}\right)\left(\beta_{j}^{n}-j h^{2} r\right), \quad 1 \leq j \leq N-1 .
$$

Taking into account (3.20) for $j$ and (3.21) for $j+1$, one gets that left hand side of (3.19) satisfies

$$
u_{j+1}^{n+1}-u_{j}^{n+1} \geq\left(1-\frac{k}{2 h^{2}}\left(\beta_{j+1}^{n}+\beta_{j}^{n}\right)-\frac{k r}{2}\right)\left(u_{j+1}^{n}-u_{j}^{n}\right), \quad 1 \leq j \leq N-2 .
$$

Let us denote

$$
\beta_{\max }=\frac{\sigma^{2} b^{2}}{[1-\eta]^{2}} .
$$

From (3.15), $\beta_{j}^{n} \leq \beta_{\max }$, and from (3.18) and (3.22) one gets

$$
1-\frac{k}{2 h^{2}}\left(\beta_{j+1}^{n}+\beta_{j}^{n}\right)-\frac{k r}{2} \geq 1-\frac{k}{h^{2}} \beta_{\max }-\frac{k r}{2} \geq 0,
$$


and taking $0<k<\frac{1}{r}$, from (3.19) it follows that

$$
u_{j+1}^{n+1}-u_{j}^{n+1} \geq 0, \quad 1 \leq j \leq N-2 .
$$

Let $j=0$, taking into account (2.7) and (3.21) for $j=1$ it follows

$$
\begin{aligned}
u_{1}^{n+1}-u_{0}^{n+1} & =u_{1}^{n+1}-(1-k r) u_{1}^{n}+(1-k r)\left(u_{1}^{n}-u_{0}^{n}\right) \\
& \geq\left(1-\frac{k}{2 h^{2}} \beta_{1}^{n}-\frac{k r}{2}\right)\left(u_{1}^{n}-u_{0}^{n}\right) \\
& \geq\left(1-\frac{k}{2 h^{2}} \beta_{\max }-\frac{k r}{2}\right)\left(u_{1}^{n}-u_{0}^{n}\right) \geq 0 .
\end{aligned}
$$

Let $j=N-1$, taking into account (2.8) and (3.20) for $j=N-1$, one gets, in an analogous way,

$$
u_{N}^{n+1}-u_{N-1}^{n+1} \geq\left(1+\frac{N k r}{2}-\left(\frac{k}{2 h^{2}} \beta_{\max }+\frac{k r}{2}\right)\right)\left(u_{N}^{n}-u_{N-1}^{n}\right) \geq 0 .
$$

Thus the result is established.

Corollary1. Under hypotheses and notation of theorem 1, and assuming that the payoff function $f(S)$ is non decreasing and nonnegative with $f(0)=$ 0 , then the scheme $(2.4),(2.7),(2.8)$ is nonnegative and nondecreasing in variable $j$ for each time stage $n$, i.e.,

$$
0=u_{0}^{n} \leq u_{1}^{n} \leq \cdots \leq u_{j}^{n} \leq u_{j+1}^{n} \leq \cdots \leq u_{N}^{n}, \quad 0 \leq n \leq \ell .
$$

For the sake of clarity in the presentation of the next stability result, we introduce a definition of stability.

Definition2. The numerical scheme (2.9)-(2.13) for the initial value problem (1.3) is said to be \|\|$_{\infty}$-stable in the fixed station sense in the domain $[0, b] \times$ $[0, T]$, if given $\tau$ with $0<\tau \leq T$, for every partition with $k=\triangle \tau, h=\triangle S$, with $\tau=\ell k$, and every $N$ with $N h=b$ one gets

$$
\left\|U^{n}\right\|_{\infty} \leq C
$$

where \|\|$_{\infty}$ denotes the supremum norm in $\mathbb{R}^{N+1}$ and $C$ is independent of $h, k$ and $N$. 
Theorem 2. Under conditions of theorem 1, the numerical scheme (2.9)(2.13) for solving initial value problem (1.3) with a convex nondecreasing and nonnegative piecewise linear payoff function is $\|\cdot\|_{\infty}$-stable.

Proof. From (2.4) for $j=N-1$ and (2.8) it follows that

$$
U_{N}^{n+1}-U_{N-1}^{n+1}=U_{N}^{n}-U_{N-1}^{n}-\frac{k}{2} \triangle_{N-1}^{n}\left(\beta_{N-1}^{n}-h^{2} r(N-1)\right), \quad 0 \leq n \leq \ell-1 .
$$

Note that from (3.16) one has $\beta_{N-1}^{n}-h^{2} r(N-1) \geq 0$ and from Lemma 1 -(i) $\triangle_{N-1}^{n} \geq 0$. Hence, from (3.27) one gets

$$
U_{N}^{n+1}-U_{N-1}^{n+1} \leq U_{N}^{n}-U_{N-1}^{n}, \quad 0 \leq n \leq \ell-1
$$

From (2.8) and (3.28) it follows that

$$
\begin{aligned}
U_{N}^{n+1} & =(1-k r) U_{N}^{n}+k r N\left(U_{N}^{n}-U_{N-1}^{n}\right) \\
& \leq(1-k r) U_{N}^{n}+k r N\left(U_{N}^{0}-U_{N-1}^{0}\right), \quad 0 \leq n \leq \ell-1 .
\end{aligned}
$$

Hence, inductively one gets

$$
U_{N}^{n} \leq(1-k r)^{n} U_{N}^{0}+N k r\left(U_{N}^{0}-U_{N-1}^{0}\right)\left(1+(1-k r)+\cdots+(1-k r)^{n-1}\right)
$$
Taking $0<k<\frac{1}{r}$, from (3.30) and using that $\sum_{j \geq 0}(1-k r)^{j}=\frac{1}{k r}$, it follows

$$
U_{N}^{n} \leq U_{N}^{0}+\left(U_{N}^{0}-U_{N-1}^{0}\right) N
$$

Note that if $s(f)$ is the slope of the last linear piece of the payoff function then

$$
U_{N}^{0}-U_{N-1}^{0}=s(f) h,
$$

and from (3.31), (3.32) and corollary 1, one gets

$$
\left\|U^{n}\right\|_{\infty}=U_{N}^{n} \leq f(N h)+s(f) h N=f(b)+s(f) b, \quad 1 \leq n \leq \ell .
$$

Thus the result is established. 


\section{Consistency}

Consistency of a numerical scheme with respect to a partial differential equation means that the exact theoretical solution of the PDE approximates well the exact theoretical solution of the difference scheme as the stepsize discretization tends to zero, [22]. This property is the objective of present section.

Let us write the scheme (2.4) in the form

$$
F\left(U_{j}^{n}\right)=\frac{U_{j}^{n+1}-U_{j}^{n}}{k}-\frac{1}{2} \beta_{j}^{n}(U) \Delta_{j}^{n}(U)-r S_{j} \nabla_{j}^{n}(U)+r U_{j}^{n}=0,
$$

where discrete operators $\nabla_{j}^{n}$ and $\Delta_{j}^{n}$ are given by (2.1) and coefficients $\beta_{j}^{n}(U)$ are given by (2.5)-(2.6). In accordance with [22, pag.100], the scheme (4.1) is said to be consistent with

$$
\begin{gathered}
L(u)=\frac{\partial u}{\partial \tau}(S, \tau)-\frac{\sigma^{2} S^{2}}{2\left(1-\lambda(S, T-\tau) S \frac{\partial^{2} u}{\partial S^{2}}(S, \tau)\right)^{2}} \frac{\partial^{2} u}{\partial S^{2}}(S, \tau) \\
-r S \frac{\partial u}{\partial S}(S, \tau)+r u(S, \tau)=0,
\end{gathered}
$$

if the local truncation error

$$
T_{j}^{n}(u)=F\left(u_{j}^{n}\right)-L\left(u_{j}^{n}\right),
$$

satisfies

$$
T_{j}^{n}(u) \rightarrow 0, \quad \text { as } \quad h=\Delta S \rightarrow 0, \quad k=\Delta \tau \rightarrow 0,
$$

where $u_{j}^{n}$ denotes the value of the analytical solution of $L(u)=0$ at the mesh point $\left(S_{j}, \tau^{n}\right), S_{j}=j h, \tau^{n}=n k$.

Let us assume that $u$ admits four times continuous partial derivatives with respect to $S$ and twice continuous partial derivatives with respect to $\tau$. Using Taylor's expansions about $\left(S_{j}, \tau^{n}\right)$ one gets

$$
\nabla_{j}^{n}(u)=\frac{\partial u}{\partial S}\left(S_{j}, \tau^{n}\right)+h^{2} E_{j}^{n}(1)
$$

where

$$
E_{j}^{n}(1)=\frac{1}{6} \frac{\partial^{3} u}{\partial S^{3}}\left(\xi_{1}, \tau^{n}\right), \quad S_{j}-h<\xi_{1}<S_{j}+h
$$


with

$$
\begin{gathered}
\left|E_{j}^{n}(1)\right| \leq \frac{1}{6} \max \left\{\left|\frac{\partial^{3} u}{\partial S^{3}}\left(S, \tau^{n}\right)\right| ; 0 \leq S \leq b\right\}=\frac{1}{6}\left|W^{n}(1)\right|_{\max } . \\
\triangle_{j}^{n}(u)=\frac{\partial^{2} u}{\partial S^{2}}\left(S_{j}, \tau^{n}\right)+h^{2} E_{j}^{n}(2)
\end{gathered}
$$

where

$$
\begin{gathered}
E_{j}^{n}(2)=\frac{1}{12} \frac{\partial^{4} u}{\partial S^{4}}\left(\xi_{2}, \tau^{n}\right), \quad S_{j}-h<\xi_{2}<S_{j}+h \\
\left|E_{j}^{n}(2)\right| \leq \frac{1}{12} \max \left\{\left|\frac{\partial^{4} u}{\partial S^{4}}\left(S, \tau^{n}\right)\right| ; \quad 0 \leq S \leq b\right\}=\frac{1}{12}\left|W^{n}(2)\right|_{\max } \\
\frac{u_{j}^{n+1}-u_{j}^{n}}{k}=\frac{\partial u}{\partial \tau}\left(S_{j}, \tau^{n}\right)+k E_{j}^{n}(3) \\
E_{j}^{n}(3)=\frac{1}{2} \frac{\partial^{2} u}{\partial \tau^{2}}\left(S_{j}, \tau\right), \quad \tau^{n}<\tau<\tau^{n+1} \\
\left|E_{j}^{n}(3)\right| \leq \frac{1}{2} \max \left\{\left|\frac{\partial^{2} u}{\partial \tau^{2}}\left(S_{j}, \tau\right)\right| ; \quad \tau^{n} \leq \tau \leq \tau^{n+1}\right\}=\frac{1}{2}\left|W_{j}^{n}(3)\right|_{\max } .
\end{gathered}
$$

Hence,

$$
\begin{aligned}
& T_{j}^{n}(u)=k E_{j}^{n}(3)-r S_{j} E_{j}^{n}(1) h^{2} \\
& -\frac{1}{2}\left(\beta_{j}^{n}(u) \triangle_{j}^{n}(u)-\frac{\sigma^{2} S_{j}^{2}}{\left(1-\lambda\left(S_{j}, T-\tau^{n}\right) S_{j} \frac{\partial^{2} u}{\partial S^{2}}\left(S_{j}, \tau^{n}\right)\right)^{2}} \frac{\partial^{2} u}{\partial S^{2}}\left(S_{j}, \tau^{n}\right)\right),
\end{aligned}
$$

where $\beta_{j}^{n}(u)$ denotes the coefficient defined as in (2.5)-(2.6),

$$
\begin{gathered}
\beta_{j}^{n}(u)=\frac{\sigma^{2} S_{j}^{2}}{\left[1-\nu_{j}^{n}(u)\right]^{2}}, \\
\nu_{j}^{n}(u)= \begin{cases}\gamma\left(1-e^{-\beta n k}\right) \triangle_{j}^{n}(u) & \text { if } \underline{S} \leq S_{j} \leq \bar{S}, \\
0, & \text { otherwise. }\end{cases}
\end{gathered}
$$


Let us introduce the function

$$
G(S, \tau, x)=\frac{x}{(1-\lambda(S, T-\tau) S x)^{2}},
$$

that in the domain

$$
\lambda(S, T-\tau) S x<1,
$$

is continuously differentiable with respect to the variable $x$, and

$$
\frac{\partial G(S, \tau, x)}{\partial x}=\frac{1+\lambda(S, T-\tau) S x}{(1-\lambda(S, T-\tau) S x)^{3}},
$$

If $\delta_{0}$ is a number with $\delta_{0}>0$ and satisfying inequality (39) of [16], take $h_{0}>0$ small enough so that

$$
\delta_{0}-\frac{h_{0}^{2}}{12}\left|W^{n}(2)\right|_{\max } \gamma\left(1-e^{-\beta \tau^{n}}\right)=\delta_{1}^{n}>0 .
$$

Let $x=\frac{\partial^{2} u}{\partial S^{2}}\left(S_{j}, \tau^{n}\right)$ and $\triangle x=h^{2} E_{j}^{n}(2)$, for $0<h<h_{0}$, then by (39) of [16] $x$ lies in the domain (4.18) and from (4.8), (4.10) and (4.20), $x+\triangle x=\triangle_{j}^{n}(u)$ lies in (4.18). Hence and using (2.5)-(2.6) and the mean value theorem, it follows the existence of some $\theta$ with $0<\theta<1$, so that

$$
\begin{aligned}
& \beta_{j}^{n}(u) \triangle_{j}^{n}(u)-\frac{\sigma^{2} S_{j}^{2}}{\left(1-\lambda\left(S_{j}, T-\tau^{n}\right) S_{j} \frac{\partial^{2} u}{\partial S^{2}}\left(S_{j}, \tau^{n}\right)\right)^{2}} \frac{\partial^{2} u}{\partial S^{2}}\left(S_{j}, \tau^{n}\right) \\
& =\sigma^{2} S_{j}^{2}\left[\frac{1+\lambda\left(S_{j}, T-\tau^{n}\right) S_{j}\left(\frac{\partial^{2} u}{\partial S^{2}}\left(S_{j}, \tau^{n}\right)+\theta h^{2} E_{j}^{n}(2)\right)}{\left(1-\lambda\left(S_{j}, T-\tau^{n}\right) S_{j}\left(\frac{\partial^{2} u}{\partial S^{2}}\left(S_{j}, \tau^{n}\right)+\theta h^{2} E_{j}^{n}(2)\right)\right)^{3}}\right] E_{j}^{n}(2) h^{2} .
\end{aligned}
$$

Let us denote

$$
\begin{gathered}
\left|W^{n}(4)\right|_{\max }=\max \left\{\left|\frac{\partial^{2} u}{\partial S^{2}}\left(S, \tau^{n}\right)\right| ; 0 \leq S \leq b\right\} \\
C(n, h)=\frac{\sigma^{2} b^{2}}{12\left(\delta_{1}^{n}\right)^{3}}\left[1+\gamma\left(1-e^{-\beta \tau^{n}}\right)\left(\left|W^{n}(4)\right|_{\max }+\frac{h^{2}}{12}\left|W^{n}(2)\right|_{\max }\right)\right] .
\end{gathered}
$$


From (4.3) and the previous considerations it follows that

$$
\left|T_{j}^{n}(u)\right| \leq\left(\frac{r b}{6}\left|W^{n}(1)\right|_{\max }+C(n, h)\left|W^{n}(2)\right|_{\max }\right) h^{2}+\frac{1}{2}\left|W_{j}^{n}(3)\right|_{\max } k .
$$

Summarizing the following result has been established:

Theorem 3. The numerical scheme (2.4)-(2.6) is consistent with equation (4.2), and the local truncation error $T_{j}^{n}$ satisfies

$$
T_{j}^{n}(u)=O\left(h^{2}\right)+O(k) .
$$

\section{Examples}

In this section we check the properties of the proposed numerical scheme (2.4)-(2.8) for the model (1.3). Furthermore, simulations are performed for different values of the price impact coefficient $\gamma$.

First example shows a situation where the numerical solution of an European vanilla call option problem behaves well under the stability conditions given in Theor. 1 and also that if such conditions are not satisfied, then they appear spurious oscillations in the numerical solution.

Example 1. Consider the vanilla call option (so that $f(s)=\max (S-E, 0)$ ) for an illiquid market with Strike price $E=50, r=0.06, \sigma=0.4, T=1$, $\underline{S}=20, \bar{S}=80, \beta=100, \gamma=1$ and $h=2$.

Figure 1 shows the option pricing value. For $k=1.4286 \cdot 10^{-4}$ the sufficient stability conditions are satisfied, dot line. For $k=7.0671 \cdot 10^{-4}$ the stability conditions are broken appearing spurious oscillations in the numerical solution, continuous line.

In the next example we consider the problem treated in example 1 under stability step size requirements but with several values of the price impact coefficient $\gamma$ simulating the illiquidity influence in the price of the option.

Example 2. Figure 2 shows the variation of the option price with the parameter $\gamma$, showing that price grows with the value of $\gamma$, mainly in the proximities of the strike price.

Figure 3 shows the variation of the numerical Delta and Figure 4 the variation of the numerical Gamma 
We notice in Figure 3 that the hedge ratio is increasing in $\gamma$ for $S<E$, and decreasing in $\gamma$ for $S>E$, i.e. lower liquidity spreads out the hedge ratio. One can see in Figure 4 that the Gamma flattens out as illiquidity increase moving its peak more and more to smaller values of $S$ and the maximum value of Gamma is reduced.

\section{Conclusions}

The fully nonlinear parabolic problem (1.3)-(1.4) appearing in [14] provides a good model for option pricing in illiquid markets by examining how price impact in the underlying asset affects the replication of a European contingent claim. In this paper we have constructed a consistent explicit finite-difference numerical scheme for (1.3)-(1.4). A sufficient condition for the discretization stepsizes has been obtained in order to guarantee positivity of the solution as well as stability for general convex payoff functions. Our scheme prevents spurious oscillations in the numerical solution because is monoticity-preserving. The numerical scheme provides desired nondecreasing solutions in the underlying asset starting from nondecreasing payoff function. Some simulations are performed to study the influence of illiquidity in the option price and the Greeks of the option.

\section{Acknowledgements}

This paper has been supported by the Spanish Department of Science and Education grant TRA2007-68006-C02-02 and the Generalitat Valenciana grant GVPRE/2008/092.

\section{References}

[1] J. Ankudinova, M. Ehrhardt, On the numerical solution of nonlinear Black-Scholes equations, Computers \& Mathematics with Applications 56 (3) (2008) 799-812.

[2] K. Back, Asymmetric information and options, Review of Financial Studies 6 (1993) 435-472.

[3] D. Bertsimas, A. Lo, Optimal control of execution costs, Journal of Financial Markets 1 (1998) 1-50. 
[4] M. Briani, R. Natalini, G. Russo, Implicit-explicit numerical schemes for jump-diffusion processes, Calcolo 44 (2007) 33-57.

[5] R. Company, L. Jódar, J.-R. Pintos, A numerical method for European option pricing with transaction costs nonlinear equation, Mathematical and Computer Modelling 50 (2009) 910-920.

[6] D. Cuoco, J. Cvitanic, Optimal consumption choices for a large investor, Journal of Economic Dynamics and Control 22 (1998) 401-436.

[7] J. Cvitanic, J. Ma, Hedging options for a large investor and forwardbackward SDEs, Annals of Applied Probability 6 (1996) 370-398.

[8] B. Düring, M. Fournier, A. Jüngel, Convergence of a high order compact finite difference scheme for a nonlinear Black-Scholes equation, Esaim-Mathematical Modelling and Numerical Analysis-Modelisation Mathematique et Analyse Numerique 38 (2004) 359-369.

[9] M. Ehrhardt, R. Mickens, A fast, stable and accurate numerical method for the Black-Scholes equation of american options, Int. J. Theor. Appl. Finance 11 (2008) 471-501.

[10] R. Frey, Market illiquidity as a source of model risk in dynamic hedging, Model Risk (2000) 125-136.

[11] R. Frey, P. Patie, Risk management for derivatives in illiquid markets: A simulation study, Advances in Finance and Stochastics (2002) 137-159.

[12] J. C. Hull, Options, Futures and Other Derivatives, 5th ed., Prentice Hall, New Jersey, 2002.

[13] P. Jorion, Value at Risk, McGraw-Hill, New York, 2000.

[14] R. Kangro, R. Nicolaides, Far field boundary conditions for BlackScholes equations, SIAM Journal on Numerical Analysis 38 (4) (2000) $1357-1368$.

[15] A. Kyle, Continuous auctions and insider trading, Econometrica 53 (1985) 1315-1335.

[16] H. Liu, J. Yong, Option pricing with an illiquid underlying asset market, J.Economic Dynamics and Control 29 (2005) 2125-2156. 
[17] J. Ma, J. Yong, Forward Backward Stochastic Differential Equations and Their Applications, Springer, New York, 1999.

[18] D. Pooley, P. Forsyth, K. Vetzal, Numerical convergence properties of option pricing PDEs with uncertain volatility, IMA J. Num. Anal. 23 (2003) 241-267.

[19] A. A. Samarskii, The Theory of Difference Schemes, Dekker, New York, 2001.

[20] W. F. Sharpe, G. J. Alexander, J. V. Bailey, Investments, Prentice Hall, New Jersey, 1999.

[21] R. Sircar, G. Papanicolaou, General Black-Scholes models accounting for increased market volatility from hedging strategies, Applied Mathematical Finance 5 (1998) 45-82.

[22] G. D. Smith, Numerical solution of partial differential equations: finite difference methods, 3rd ed., Clarendon Press, Oxford, 1985.

[23] D. Vayanos, Strategic trading in a dynamic noisy market, Journal of Finance 56 (2001) 131-171.

[24] D. Ševčovič, An iterative algorithm for evaluating approximations to the optimal exercise boundary for a nonlinear Black-Scholes equation, Canad. Appl. Math. Quarterly 15 (1) (2007) 77-97.

[25] D. Ševčovič, Nonlinear Models In Mathematical Finance: New Research Trends In Option Pricing. In: M. Ehrhardt (ed.), chap. Transformation methods for evaluating approximations to the optimal exercise boundary for linear and nonlinear Black-Scholes equations, Nova Science Publishers, New York, 2008, pp. 173-218.

Figure 1: Numerical solutions for several temporal step size discretization values. 
Figure 2: Variation of the option price with the parameter $\gamma$.

Figure 3: Variation of the Delta.

Figure 4: Variation of the Gamma. 